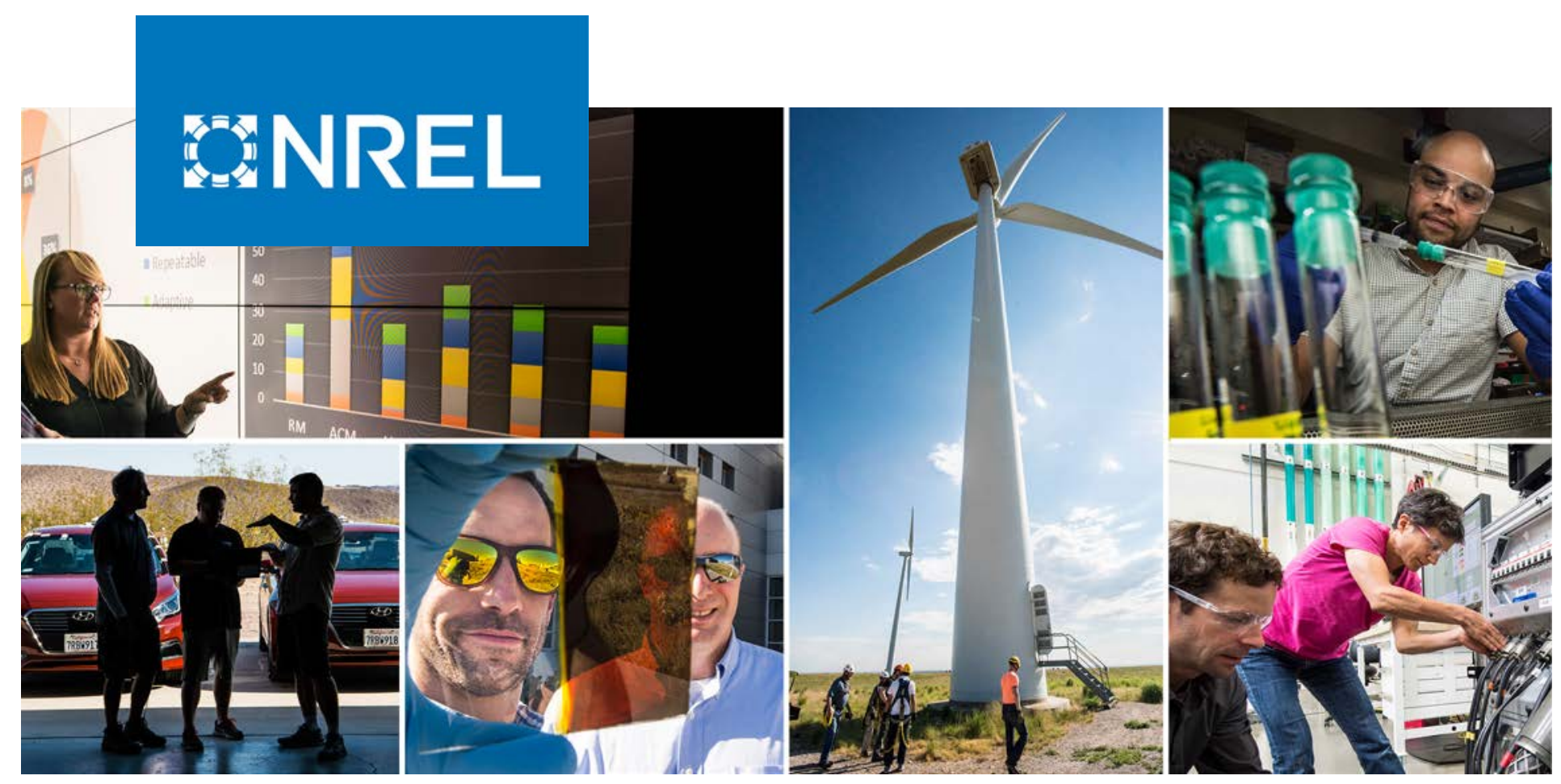

\title{
Power and Communications Hardware- in-the-Loop CPS Architecture and Platform for DER Monitoring and Control Applications
}

\section{Preprint}

Jianhua Zhang, ${ }^{1}$ Kumaraguru Prabakar, ${ }^{2}$

Adarsh Hasandka, ${ }^{2}$ S M Shafiul Alam, ${ }^{3}$ Yazhou Jiang, ${ }^{1}$

Bri-Mathias Hodge, ${ }^{2,4}$ and David Wenzhong $\mathrm{Gao}^{5}$

1 Clarkson University

2 National Renewable Energy Laboratory

3 Idaho National Laboratory

4 University of Colorado, Boulder

5 University of Denver

Presented at the 2021 IEEE International Conference on Communications, Control, and Computing Technologies for Smart Grids (SmartGridComm) October 25-28, 2021

NREL is a national laboratory of the U.S. Department of Energy

Office of Energy Efficiency \& Renewable Energy

Operated by the Alliance for Sustainable Energy, LLC

This report is available at no cost from the National Renewable Energy Laboratory (NREL) at www.nrel.gov/publications.
Conference Paper NREL/CP-5D00-78433 January 2022 


\title{
WNREL
}

\section{Power and Communications Hardware- in-the-Loop CPS Architecture and Platform for DER Monitoring and Control Applications}

\section{Preprint}

\author{
Jianhua Zhang, ${ }^{1}$ Kumaraguru Prabakar, ${ }^{2}$ \\ Adarsh Hasandka, ${ }^{2}$ S M Shafiul Alam, ${ }^{3}$ Yazhou Jiang, ${ }^{1}$ \\ Bri-Mathias Hodge, ${ }^{2,4}$ and David Wenzhong Gao ${ }^{5}$ \\ 1 Clarkson University \\ 2 National Renewable Energy Laboratory \\ 3 Idaho National Laboratory \\ 4 University of Colorado, Boulder \\ 5 University of Denver
}

\author{
Suggested Citation \\ Zhang, Jianhua, Kumaraguru Prabakar, Adarsh Hasandka, S M Shafiul Alam, Yazhou \\ Jiang, Bri-Mathias Hodge, and David Wenzhong Gao. 2022. Power and Communications \\ Hardware-in-the-Loop CPS Architecture and Platform for DER Monitoring and Control \\ Applications: Preprint. Golden, CO: National Renewable Energy Laboratory. NREL/CP- \\ 5D00-78433. https://www.nrel.gov/docs/fy21osti/78433.pdf.
}

(C) 2022 IEEE. Personal use of this material is permitted. Permission from IEEE must be obtained for all other uses, in any current or future media, including reprinting/republishing this material for advertising or promotional purposes, creating new collective works, for resale or redistribution to servers or lists, or reuse of any copyrighted component of this work in other works.

NREL is a national laboratory of the U.S. Department of Energy Office of Energy Efficiency \& Renewable Energy Operated by the Alliance for Sustainable Energy, LLC

This report is available at no cost from the National Renewable Energy Laboratory (NREL) at www.nrel.gov/publications.

Contract No. DE-AC36-08GO28308
Conference Paper

NREL/CP-5D00-78433

January 2022

National Renewable Energy Laboratory 15013 Denver West Parkway Golden, CO 80401

303-275-3000 • www.nrel.gov 


\section{NOTICE}

This work was authored in part by the National Renewable Energy Laboratory, operated by Alliance for Sustainable Energy, LLC, for the U.S. Department of Energy (DOE) under Contract No. DE-AC36-08GO28308. Funding provided by the U.S Department of Energy Office of Energy Efficiency and Renewable Energy Solar Energy Technologies Office. The views expressed herein do not necessarily represent the views of the DOE or the U.S. Government.

This report is available at no cost from the National Renewable Energy Laboratory (NREL) at www.nrel.gov/publications.

U.S. Department of Energy (DOE) reports produced after 1991 and a growing number of pre-1991 documents are available free via www.OSTI.gov.

Cover Photos by Dennis Schroeder: (clockwise, left to right) NREL 51934, NREL 45897, NREL 42160, NREL 45891, NREL 48097, NREL 46526.

NREL prints on paper that contains recycled content. 


\title{
Power and Communications Hardware-In-the-Loop CPS Architecture and Platform for DER Monitoring and Control Applications
}

\author{
Jianhua Zhang ${ }^{1}$, Kumaraguru Prabakar ${ }^{2}$, Adarsh Hasandka ${ }^{2}$, S M Shafiul Alam ${ }^{3}$, \\ Yazhou Jiang ${ }^{1}$, Bri-Mathias Hodge ${ }^{2,4}$, and David Wenzhong Gao $^{5}$ \\ ${ }^{1}$ Department of Electrical and Computer Engineering, Clarkson University, Potsdam, NY 13699, USA \\ ${ }^{2}$ National Renewable Energy Laboratory (NREL), Golden, CO 80401, USA \\ ${ }^{3}$ Idaho National Laboratory (INL), Idaho Falls, ID 83415, USA \\ ${ }^{4}$ Department of Electrical and Computer Engineering, The University of Colorado, Boulder, Colorado 80309, USA \\ ${ }^{5}$ Department of Electrical and Computer Engineering, University of Denver, Denver, CO 80208, USA \\ Email: \{jzhang, yjiang\}@ clarkson.edu, \{kumaraguru.prabakar, Adarsh.Hasandka, Bri.Mathias.Hodge\}@ nrel.gov, \\ SMShafiul.Alam@inl.gov, David.Gao@du.edu
}

\begin{abstract}
The rapid growth of distributed energy resources (DERs) has prompted increasing interest in the monitoring and control of DERs through hybrid smart grid communications. The deployment of communications and computation has transformed the traditional physical power grid into a smart cyberphysical system (CPS). To fully understand the interdependence of physical grid and cyber networks, this study designed a power and communications hardware-in-the-loop (PCommHIL) CPS architecture. This architecture enables the flexible verification of DER monitoring and control with hybrid communications architectures and internet protocols. Design, development and case study of a PCommHIL testbed for the DER coordination are discussed in detail, and the proposed platform integrates DER devices, advanced metering infrastructures (AMIs), and a suite of hybrid communications network for distribution automation applications. Case study on DER situational awareness and VoltVar control validates the efficacy of this proposed PCommHIL platform with hybrid communications designs. Results show that the home area network (HAN) communication technologies play a critical role in hybrid designs and it is the bottleneck for DER applications. High performance communication technologies are highly recommended to be applied in the HAN for enhanced monitoring and real-time control of DERs.

Index Terms-Controller hardware-in-the-loop (CHIL), cyberphysical system (CPS), digital real time simulation (DRTS), distributed energy resources (DER), hardware-in-the-loop (HIL), power hardware-in-the-loop (PHIL).
\end{abstract}

\section{INTRODUCTION}

Increasing integration of distributed energy resources (DERs) into distribution grids has spurred great interest in

This work was authored by the National Renewable Energy Laboratory, operated by Alliance for Sustainable Energy, LLC, for the U.S. Department of Energy (DOE) under Contract No. DE-AC36-08GO28308. Funding is provided by U.S. Department of Energy Office of Energy Efficiency and Renewable Energy Solar Energy Technologies Office. The views expressed in the article do not necessarily represent the views of the DOE or the U.S. Government. The U.S. Government retains and the publisher, by accepting the article for publication, acknowledges that the U.S. Government retains a nonexclusive, paid-up, irrevocable, worldwide license to publish or reproduce the published form of this work, or allow others to do so, for U.S. Government purposes. the DER management system (DERMS) for both distribution system operators (DSOs) and transmission system operators (TSOs) [1]-[3]. DERs refer to distributed renewable generations, e.g. rooftop solar photovoltaic (PV) panels, small wind turbines, residential battery storage systems, electric vehicles (EV), EV charging stations (EVCS), and controllable loads. The emerging concept of smart distribution network indicate that DERs have high potential to support grid operations in two ways: a) optimizing energy performance of DERs to address stochastic and dynamic operational challenges of power grids; b) supporting grid operation by providing frequency regulation service and voltage support [4]. However, all these advanced applications of smart distribution systems rely heavily on new control, protection and communication systems of DERs [5]. Field testing of new technologies is prohibitive for utilities even though it has high value. Alternatively, the hardware-inthe-loop (HIL) technique with both grid systems and communications networks provides an effective means to mimic and evaluate the real-world grid operation with DERs under various scenarios and events.

HIL CPS testbed technology, as a promising solution, has been studied in the research community to investigate the interactions among different infrastructures for DER applications [6]-[11]. Specifically, the increasing prevalence of DERbased CPS and their common-mode vulnerabilities may lead to cyber-threats and risk of tripping DERs offline, resulting in power disruptions and instability in the distribution grid operation. The HIL DER testbeds, such as power HIL [6], [7], communications HIL [8], power and controller HIL [9], have been developed with separate and dedicated HIL cosimulation approaches. Several existing DER CPS testbeds have been reviewed in [10]. One example HIL testbed is the NREL DER test facility [11], which mainly focuses on characterizing the performance and reliability of DER systems, supporting standards development, and investigation on emerging \& complex system integration challenges. The 
IEEE 1547 standards for the DER interconnection have also been developed and validated in this DER test facility [12]. However, the validation of DER applications on the holistic HIL CPS testbed remains under-investigated.

Built upon these facilities at NREL, this study further proposes a power and communications hardware-in-the-loop (PCommHIL) CPS testbed for characterizing the performance and reliability of the DER system. It aims to meet the increasing needs of simulation of the interconnected physical power grid, communication infrastructure, and emerging DERMS applications under one holistically integrated platform. The main contributions of this paper are threefold: 1)industry-grade DER and smart meter hardwares, hybrid communications network, and DERMS, have been developed and integrated into the proposed PCommHIL CPS testing platform, which provides an effective means to validate advanced DER applications; 2) two DER monitoring and control cases of grid applications have been developed via hybrid communications network at the utility-scale distribution grid. Hybrid communications network refer to the network with more than two different communication technologies within one network. and 3) a suite of hybrid communications network designs have been investigated to assess the impact on latency and packet loss rate for DER monitoring. Even though this study only considers two applications, the proposed testing platform has the flexibility and scalability for other smart grid cyber-physical and cyber-security applications.

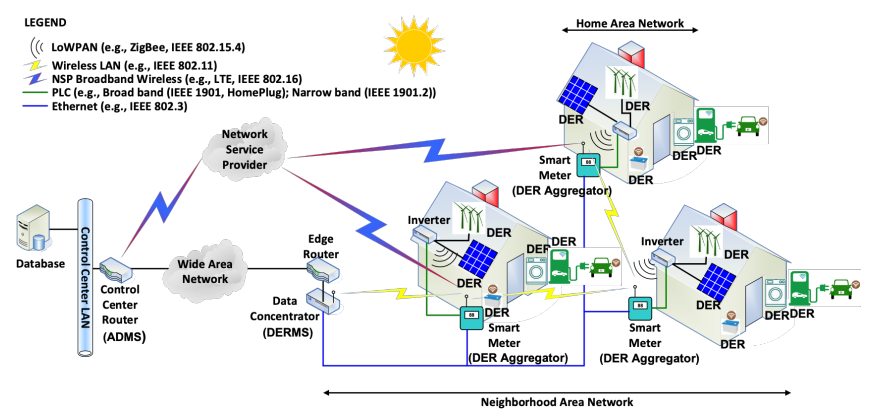

Fig. 1. DER Applications Architecture with Hybrid Communications

\section{Architecture Design of Power And COMMUNICATIONS HIL CPS TESTBED}

In this section, the hybrid communications system and the corresponding architecture design of the PCommHIL testing platform are presented in detail.

\section{A. DER Monitoring and Control Architecture with Hybrid Communications System}

The hybrid communications system for DER monitoring and control typically consists of three subnetwork as shown in Fig. 1: 1) Home area network (HAN), where DER devices are interconnected and the smart meter works as the DER aggregator; In HAN, usually low-power wireless personal area network (LoWPAN), Zigbee and power line communication (PLC) are used; 2) Neighborhood area network (NAN), by which the DER monitoring information and control signals are exchanged between smart meters at the customer's premise and the local data concentrators (DC); Commonly used in NAN are three communication technologies: WiFi ad-hoc, WiMAX, and Ethernet cables; Both ADMS consisting of the situational awareness of DERs, electrical modeling, and power flow functions, and DERMS including least-cost optimization control, integration, and interfaces of DERs, are usually implemented in the data concentrator of the utility control room. 3) Wide-area network (WAN), through which the data from local collectors are passed on to the control room at DSOs or TSOs for situational awareness and advanced grid applications; WAN usually employs high-speed optical cable, commercialized AT\&T and Verizon telecommunication network, or PLC in utilities. Thus, from the DER to the utility control room, a hybrid communications network consists of HAN, NAN, and WAN with different communication protocols may be used for DER applications. The four most popular alternative designs shown in Table I.

TABLE I

HYBRID COMMUNICATIONS SYSTEM FOR DER APPLICATIONS

\begin{tabular}{ccc}
\hline Hybrid Type & Home Area Network & Neighborhood Area Network \\
\hline Hybrid 1 & Zigbee & Ethernet cable \\
Hybrid 2 & Zigbee & WiFi \\
Hybrid 3 & PLC & Ethernet cable \\
Hybrid 4 & PLC & WiFi \\
\hline
\end{tabular}

\section{B. Architecture of Power and Communications HIL Testbed}

To validate the interplay among practical DER devices, communications infrastructures, and advanced grid applications, we designed the architecture of the power and communications HIL (PCommHIL) CPS testing platform with DER applications in the loop, as shown in Fig. 2. The interoperability and flexibility feature of the proposed architecture allows for: 1) DER interconnection and DER devices to be tested under realistic but simulated conditions; 2) evaluation performance of of the designed communications infrastructure and protocols; and 3) flexible evaluation of DER applications.

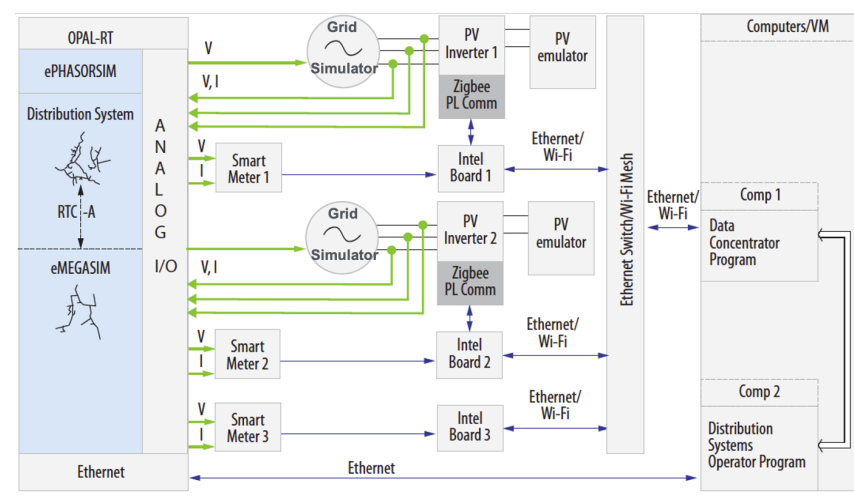

Fig. 2. Architecture Design of Power and Communications HIL Testbed

The architecture of the CPS testbed comprised of both physical and cyber subsystems is shown in Fig. 2. In the physical subsystem, a digital real-time simulator-Opal-RT-is used to simulate the utility-scale power grid, which provides simulated input data to the physical smart meters and to design DER applications in a timescale from microseconds 
(for electromagnetic transient simulations) to milliseconds (for dynamic phasor simulations). The output signals from the control application are fed back to the DER hardware and further to the power system simulator in real time. The simulation is updated based on feedback from the DER hardware, which needs to be supplied with operational power (AC power, DC power or both) that is consistent with conditions in the power grid simulation. One example of the operational power is a bidirectional AC power amplifier based on a voltage signal from the power grid simulation, referred to as a grid simulator, that has been typically used to supply the AC power required by the DER power hardware. Sensors measure the AC current supplied by the grid simulator and transmit that data to the power grid simulation as a feedback. To ensure a stable and accurate PHIL simulation, compensation needs to be added to the loop, as described in [13]. Similarly, a DC power amplifier can be controlled to deliver DC power consistent with the power system simulation when necessary.

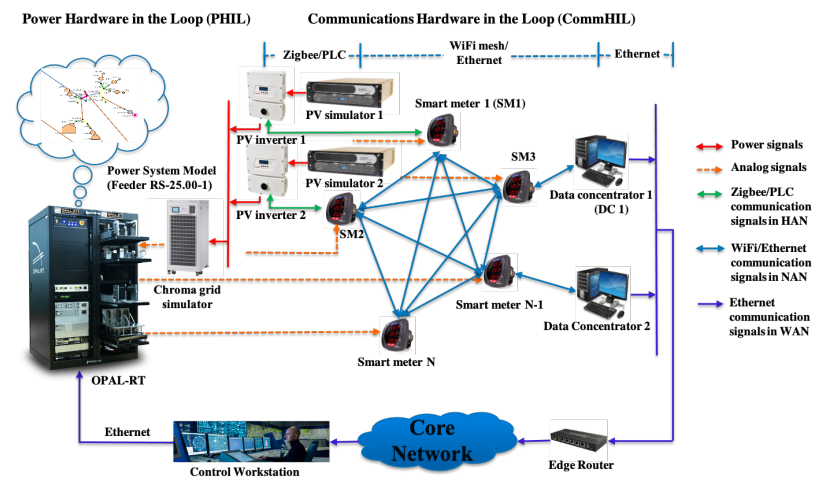

Fig. 3. Implementation Diagram of the PCommHIL CPS Testing Platform

In the proposed design, a photovoltaic (PV) inverter is connected to a DC power amplifier, referred to as a $\mathrm{PV}$ simulator, and to a Chroma grid simulator. The PV simulator is configured with the solar insolation data or it can be controlled from the Opal-RT. The inverter operates as if it were installed with solar panels and connected to a utility grid, which allows researchers to explore its behavior under a wide range of operational conditions. This emulation technique is more realistic than simulation because the simulation models may not well capture the full range of realistic behaviors. Also, it allows for testing of proprietary systems. In addition, the smart meter-based advanced measurement infrastructure (AMI) is connected to the Opal-RT through the analog signal, and it enables to capture the realistic measurement behaviors and the extended computational and communication capabilities of smart meters using the Intel single board computer enable the flexible deployment of DER applications.

In the cyber subsystem described in Subsection II-A, the HAN consists of the PV inverter and its attached smart meter through the power line communication (PLC) and Zigbee. To enable these two alternative communication technologies in the PV inverter, the Intel single board computer is also employed. The NAN consists of smart meters and the data concentrator through the most pervasive communication technologies of WiFi mesh and Ethernet cable. The data concentrator refers to the general-purpose computer which allows the flexible implementation of the DERMS and ADMS. Compared with the communication network simulator, the advantage of integrating physical hybrid communications infrastructure into the CPS testbed is to capture the full range of realistic behaviors of hybrid designs and cyber-physical DER applications

\section{IMPLEMENTATION OF POWER AND COMMUNICATION HIL CPS PLATFORM}

Following the architecture design of the proposed PCommHIL CPS testbed, the implementation diagram is shown in Fig. 3, and it consists of power/communications hardware in the loop (PHIL/CommHIL) subsystems. The platform is setup at the Energy System Integration Facility (ESIF) of National Renewable Energy Laboratory (NREL) and the physical layout is shown in Fig. 4. In the PHIL subsystem, the power components include two TerrSAS PV simulators (600V/25A), two Solar Edge single phase PV inverters (240VAC/16A), the Chroma grid simulator (60kVA), the Opal-RT simulator, and three Shark 200 smart meters. The functions and connection through power and analog signals have been described in the Subsection II-B.

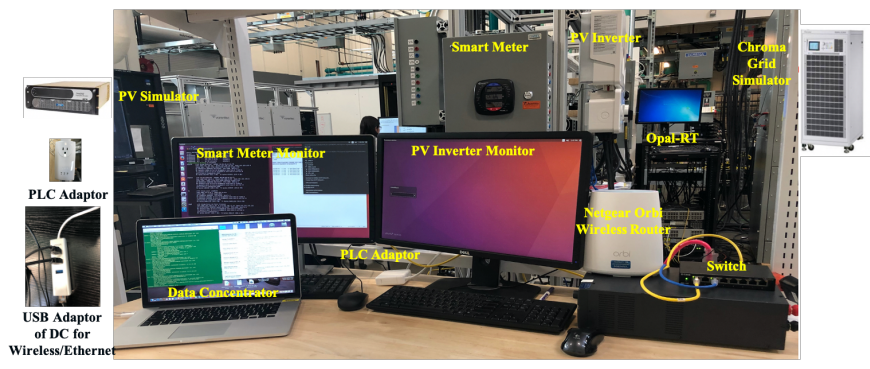

Fig. 4. Layout of the PCommHIL CPS Testing Platform at the Energy System Integration Facility (ESIF) of NREL

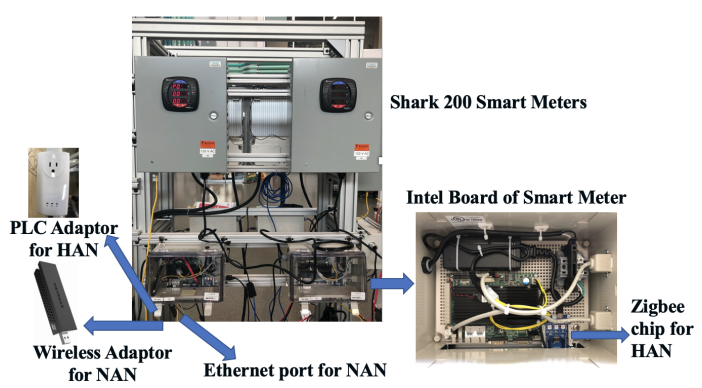

Fig. 5. Smart Meter with Communication and Computational Capability

In the CommHIL subsystem, the personal Mac laptop works as the data concentrator and the attached USB adaptor enables both Ethernet cable and WiFi connections in Fig 4. The Netgear Orbi wireless router is employed to setup the WiFi meshed NAN and the switch is used to build the Ethernet NAN. From the communication point of view, each smart meter as the joint node in both HAN and NAN, is required to support four communication technologies. The Intel single board computer is attached to each smart meter and configured 
to connect to 1) wireless adaptor and Ethernet cable for NAN, and 2) PLC adaptor and Zigbee chip for HAN as shown in Fig. 5. Similarly, each Solar Edge single phase inverter is attached with the Intel single board computer to enable connections of PLC adaptor and Zigbee chip for HAN. Note that the control command for the PV inverter can be delivered through the hybrid communications network, and the control command for simulated power modules can be transferred from data concentrator to Opal-RT through Ethernet cable, which is shown as the bright blue line in Fig. 3.

The links in the hybrid communications network is setup according to the designed IP addressing scheme. Each communication link, accuracy of the timestamp reporting, and the simultaneous use of multiple communication links are verified by sending sample packets obtained from both PV intervters and smart meters with the basic configuration. In particular, each communication node is synchronized to the Apple time server to enable the accurate timestamp. Techniques such as tuning system kernel parameters of system overhead and latency, and switching to a low latency kernel, are applied to improve the network latency.

\section{Case Study of Hybrid Communications EfFects ON DER APPLICATIONS}

To evaluate the impact of hybrid communications network on DER applications, both open-loop and closed-loop case studies have been conducted. This section presents the reference test case (RTC), digital real-time simulation model of the RTC, DER monitoring, control, and test bed results.

\section{A. Reference Test Case}

We consider the taxonomy feeder titled R2-25.00-1-referred to as the RTC-containing 1,080 nodes. More details on the feeder model is available in [14]. Fig. 6 shows the network topology of the distribution feeder used in the setup. Two PV inverters, three smart meters, and one data concentrator exist within two HANs and one NAN. These devices were integrated into the simulated model of the feeder through analog IOs.

\section{B. Development of RTC's ePHASORSIM model}

The ePHASORSIM is used to simulate the utility-scale distribution grid of RTC in the Opal-RT. The ePHASORsim's phasor-domain solver typically performs at a time step of several milliseconds, providing voltage and current information in the phasor format. This phasor domain model was interfaced to the power hardware through analog IOs. Current information from the power hardware components are fed to the ePHASORSIM model through the Simulink/RT-LAB.

This model was converted from GridLAB-D to ePHASORSIM model. The topology of the converted model is shown in in Fig. 6. Three smart meters are configured to measure the voltage magnitude and angle of Node 217, the current magnitude and angle of Node 13, and the real and reactive power of Load 65, respectively. Two PV inverters are attached to Node 217 and 13, respectively.

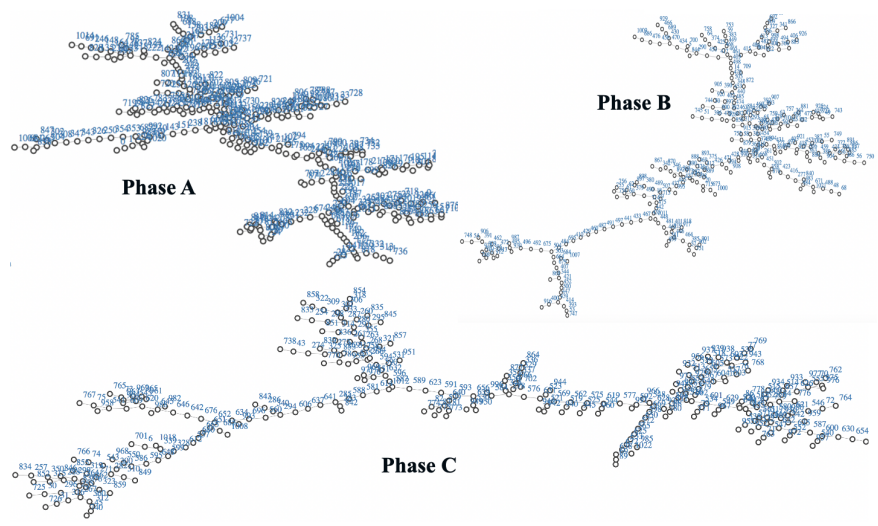

Fig. 6. Three Single-Phase Topologies of RTC in ePHASORsim

\section{Development of DER Monitoring and Control Applications}

We consider the DER monitoring and control applications to validate the efficacy of the developed PCommHIL CPS testing platform and to evaluate the performance of hybrid communications designs for grid applications. In the data concentrator, monitoring functions of the PV inverter and the smart meter, and the Volt-Var control algorithm of the PV inverter, shown in Fig. 7(a), are implemented. As Fig. 7(b) shows, the implementation consists of initialization and loop blocks and each block contains the detailed steps.

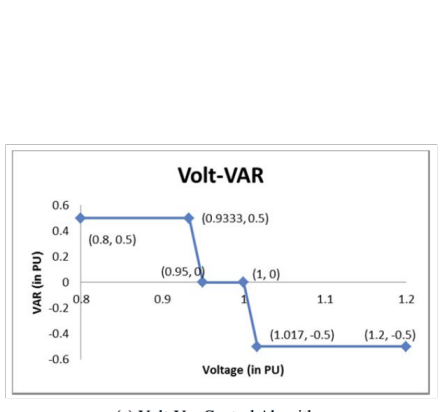

(a) Volt-Var Control Algorithm

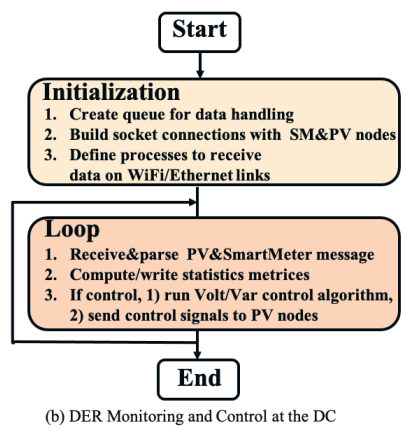

(b) DER Monitoring and Control at the DC
Fig. 7. Implementation of DER Monintoring and Control Apps at the DC

Similarly, both applications of smart meter monitoring and PV monitoring and control are implemented in the smart meter's Intel single computer. Compared with the implementation in the data concentrator of Fig. 7(b), the initialization block also includes the Modbus connection setup with the smart meter to extract grid status, and the message forwarding function for the PV monitoring and control is located in the loop block. Finally, the PV inverter's Intel single computer is employed to extract PV status data and dispatch the PV control signal with the PV inverter via the Modbus connection.

\section{Result Analysis}

We initially conducted the monitoring function test and aimed to answer three questions: 1) whether four hybrid communication network designs can meet the requirement of the latency and packet loss rate; 2) which subnetwork dominates the performance of the whole hybrid network; 
and 3) how different data traffics impact the performance of communications network. To answer these questions, three scenarios with different traffic types are considered: a) PV data traffic on four hybrid designs; b) smart meter data traffic on two types of NAN; and c) both PV and smart meter data traffics on four hybrid designs. The PV data sample rate is $0.5 \mathrm{sample} / \mathrm{s}$, and each sample with 14-byte in length includes 4-byte grid voltage, 3-byte PV inverter name, 3-byte link name, and 4-byte time stamp. Thus, the sending rate of PV data traffic is 7 bytes/s or more in practice. The smart meter sample rate is also 0.5 sample/s and each sample contains 72-byte measurement data, 3-byte smart meter name, 3-byte link name, and 4-byte time stamp, amounting to 82 bytes in length per sample measurement. The grid measurement data includes three-phase line-to-line voltage, current, real power, reactive power, apparent power, and power factor. Thus, the sending rate of smart meter data traffic is 41 bytes/s.

TABLE II

RESUlts OF DER MONITORING AT PCHIL PlatForm

\begin{tabular}{clclc}
\hline Index & Scenario & Latency (ms) & Scenario & Latency (ms) \\
\hline 1 & SM_WiFi & 6.48 & SM_Ethernet & 1.95 \\
2 & PV_Zigbee_WiFi & 1311 & PV_Zigbee_Ethernet & 839 \\
3 & PV_PLC_WiFi & 1302 & PV_PLC_Ethernet & 837 \\
4 & PVSM_Zigbee_WiFi & 1210 & PVSM_Zigbee_Ethernet & 804 \\
5 & PVSM_PLC_WiFi & 1237 & PVSM_PLC_Ethernet & 786 \\
\hline
\end{tabular}

Table II shows the latency results of monitoring function for the three scenarios at the PCommHIL platform. From the implementation results, three observations of the data package latency performance are: 1) Due to the high bandwidth of $\mathrm{WiFi}$ and Ethernet, the latency of $\mathrm{WiFi}$ and Ethernet based NANs in Scenario 2 of Table.II with smart meter data traffic are $6.48 \mathrm{~ms}$ and $1.95 \mathrm{~ms}$, respectively, even though the smart meter measurement rate of 41 bytes/s is much higher than the PV data rate of 7 bytes/s. Nevertheless, both meet the requirement of $300 \mathrm{~ms}$ of package latency. 2) From the results of Scenarios 1 and 3 involving PV data traffic, the latency performance for all four hybrid designs are around 1 second, which does not meet the requirement of $300 \mathrm{~ms}$. These results indicate that the bandwidth of HAN denominates the latency performance of hybrid network due to the lower capacity, which is consistent with the simulation results. 3) Comparing the latency performance in Scenario 1 with only PV data traffic with that in Scenario 3 with both PV and smart meter traffic, it concludes that there is no strong inter-dependency between traffic types and the latency performance because of the limited bandwidth in HAN and sufficient bandwidth in NAN. Experiment results show that the packet loss rate for all scenarios are zero, which meets the requirement of less than $0.1 \%$ packet loss rate.

\section{CONCLUSiON}

In this paper, we developed a new system-in-the-loop (SIL) for DER monitoring and control and a new power and communications hardware-in-the-loop (PCommHIL) CPS testing platform. The developed PCommHIL testing platform consists of simulated distribution grid, DER and smart meter hardware, hybrid communications infrastructure, and DERMS, which enables a greater interoperability and flexibility to accommodate different communication protocols and network. Two DER monitoring and control applications with hybrid communication infrastructures have used to validate the efficacy of the proposed PCommHIL CPS testing platform. The case study with hybrid communications designs for the DER monitoring shows that the HAN communication technologies dominates the overall performance of hybrid communications designs. Future research will focus on the development, implementation, and validation of advanced grid interactive DER dispatch algorithms, their cyber-physical fashion and further cyber-security strategies for the DER-based smart distribution grid with the developed PCommHIL CPS testing platform.

\section{REFERENCES}

[1] "The global annual market for the department of distributed solar pv plus energy storage is expected to exceed $\$ 49$ billion by 2026," May 2017. [Online]. Available: https://www.navigantresearch.com/newsroom/theglobal-annual-market-for-the-deployment-of-distributed-solar-pv-plusenergy-storage-is-expected-to-exceed-49-billion-by-2026

[2] "Understanding derms," July 2018. [Online]. Available: https://www.epri.com/research/products/3002013049

[3] "Electric program investment charge (epic) 2.02 - distributed energy resource management system," Jan. 2019. [Online]. Available: https://www.pge.com/pge_global/common/pdfs/aboutpge/environment/what-we-are-doing/electric-program-investmentcharge/PGE-EPIC-2.02.pdf

[4] D.Giustina and S. Rinaldi, "Hybrid communication network for the smart grid: Validation of a field test experience," IEEE Transcation on Power Delivery, vol. 10, no. 1, pp. 251-261, March 2015.

[5] M. Zajc, M. Kolenc, and N. Suljanovic, "Virtual power plant communication system architecture," Smart Power Distribution Systems, pp. 231-250, 2018

[6] G. Ravikumar, B. Hyder, and M. Govindarasu, "Hardware-in-the-loop cps security architecture for der monitoring and control applications," in 2020 IEEE Texas Power and Energy Conference (TPEC), 2020, pp. $1-5$.

[7] F. Huerta, J. K. Gruber, M. Prodanovic, and P. Matatagui, "A power-hil microgrid testbed: Smart energy integration lab (seil)," in IEEE Energy Conversion Congress and Exposition (ECCE), 2014, pp. 3998-4003.

[8] G. Ravikumar, B. Hyder, and M. Govindarasu, "Hardware-in-the-loop cps security architecture for der monitoring and control applications," in IEEE Texas Power and Energy Conference (TPEC), 2020, pp. 1-5.

[9] P. Kotsampopoulos, D. Lagos, N. Hatziargyriou, M. O. Faruque, G. Lauss, O. Nzimako, P. Forsyth, M. Steurer, F. Ponci, A. Monti, V. Dinavahi, and K. Strunz, "A benchmark system for hardware-in-theloop testing of distributed energy resources," IEEE Power and Energy Technology Systems Journal, vol. 5, no. 3, pp. 94-103, 2018.

[10] M. H. Cintuglu, O. A. Mohammed, K. Akkaya, and A. S. Uluagac, "A survey on smart grid cyber-physical system testbeds," IEEE Communications Surveys Tutorials, vol. 19, no. 1, pp. 446-464, 2017.

[11] NREL, "Distributed energy resources test facility," in NREL,DER, Golden, CO, USA, 2015

[12] "An overview of for distributed energy resource (der) interconnection: Current practices and emerging solutions," National Renewable Energy Laboratory, CO, USA, Technical Report, Tech. Rep., April 2019.

[13] N. Ainsworth, A. Hariri, K. Prabakar, A. Pratt, and M. Baggu, "Modeling and compensation design for a power hardware-in-the-loop simulation of an ac distribution system," in 2016 North American Power Symposium (NAPS), 2016, pp. 1-6.

[14] K. P. Schneider, Y. Chen, D. P. Chassin, R. G. Pratt, D. W. Engel, and S. E. Thompson, "Modern grid initiative distribution taxonomy final report," 11 2008. [Online]. Available: https://www.osti.gov/biblio/1040684 